\title{
Single Linkage Cluster Analysis
}

National Cancer Institute

\section{Source}

National Cancer Institute. Single Linkage Cluster Analysis. NCI Thesaurus. Code C65177.

A clustering method in which the distance between two clusters is determined by the distance of the two closest objects (nearest neighbors) in the different clusters. 